\title{
Ensayos de Captadores Flotantes Curvos para el Calentamiento de Estanques
}

\section{Heating an Aquaculture pond with a curved floating collector}

\section{Mareva Y. Gutierres S.}

Grupo de Investigación de Tecnologías Energéticas Apropiadas (GITEA), Facultad Regional Resistencia, Universidad Tecnológica Nacional - Argentina.

mareygs@gmail.com

\section{Gustavo R. Figueredo}

Grupo de Investigación de Tecnologías Energéticas Apropiadas (GITEA), Facultad Regional Resistencia, Universidad Tecnológica Nacional - Argentina.

grfigueredo@gmail.com

\section{Ruben A. Spotorno}

Grupo de Investigación de Tecnologías Energéticas Apropiadas (GITEA), Facultad Regional Resistencia, Universidad Tecnológica Nacional - Argentina.

rubenspotorno@yahoo.com

\section{Resumen}

Los peces son afectados por las temperaturas bajas del agua durante los meses invernales, lo que ocasiona disminución de la tasa de reproducción y de la producción en la acuicultura. Se efectuaron ensayos en dos modelos de estanques, en el primero se colocaron captadores flotantes y el segundo funcionó como testigo. Buscando morigerar los descensos de temperatura del agua, se ensayaron captadores solares flotantes con cubierta de acetato curva. Las experiencias fueron efectuadas bajo las condiciones climáticas del NEA, durante el invierno. El captador solar flotante tuvo una respuesta aceptable respecto al incremento de temperatura buscado. Se presentan las diferencias de temperatura entre las dos piletas de ensayo para los distintos periodos, además de otros parámetros climáticos. Se puedieron conseguir incrementos de la temperatura mínima del agua de $6,2{ }^{\circ} \mathrm{C}$ durante julio y de $8{ }^{\circ} \mathrm{C}$ durante agosto en relación con la temperatura ambiente

Palabras clave: Acuicultura, captadores solares flotantes, estanques piscicultura. 


\begin{abstract}
Fishes are affected by low temperatures of water in winter months, that causes a decrease in reproduction rate and in aquaculture production. Tests were carried out in two ponds models; floating collectors were placed in the first one and the second one worked as a reference sample. Looking to moderate temperature decrease, floating collectors with acetate-curved cover were tested. The experiences were carried out under NEA climatic conditions during winter. A good performance was obtained from floating collector in reference to the increase in temperature wanted. Differences of temperatures are presented between two ponds models. There in addition to other climatic parameters. During July, it was obtained a temperature increase of $6,2{ }^{\circ} \mathrm{C}$ and in August, the increase was $8{ }^{\circ} \mathrm{C}$ between water and atmosphere temperature.
\end{abstract}

Keywords: Aquaculture, water temperature, Heat balance.

\title{
Introducción
}

Los peces son organismos poiquilotermos cuya temperatura corporal depende del medio en que viven. Por lo tanto la temperatura es la variable más importante y determinante para el cultivo, y a su vez la más difícil de controlar, (Manual básico de Piscicultura en estanques, FAO 2010). Todas las actividades fisiológicas de los peces (respiración, digestión, excreción, alimentación, movimientos) están íntimamente ligadas a la temperatura del agua. Cuanto más alta es la temperatura, mayor es la actividad de los peces, consecuentemente disminuye el oxígeno disuelto en el agua. El oxígeno es utilizado por los peces para que la energía contenida en los alimentos pueda ser liberada y ser aprovechada para las funciones vitales; la falta de oxígeno en el agua los perjudica sensiblemente. Es por ello, que la cantidad de oxígeno disuelto es limitante para la sobrevivencia de los peces. Los encargados de producir oxígeno en un estanque son el fitoplancton y las plantas acuáticas. Esta producción tiene variantes a lo largo del día, siendo alta durante las horas de luz solar y mínima antes del amanecer. Cada especie tiene sus requerimientos óptimos de oxígeno, sin embargo, de forma general se recomienda que los valores permanezcan por encima del 75 al $80 \%$ de saturación. Cada especie posee un rango óptimo de temperatura para crecer y desarrollarse. Conocer las variaciones de la temperatura a lo largo del día, así como de una estación a otra, permitirá decidir la especie a cultivar y determinar el tipo de manejo a realizar. (Manual básico de Piscicultura en estanques, FAO 2010).

En nuestro país el pacú es encontrado en forma natural en las aguas continentales. En la actualidad, se realizan cultivos de tipo experimental y piloto comercial en las provincias de Corrientes, Misiones, Chaco, Formosa y Santa Fe, con diferentes modalidades y resultados. Las condiciones climáticas son favorables para la recría y engorde de pacú en cautiverio. En la región del NEA las temperaturas de invierno suelen ser sensiblemente bajas en algunas oportunidades, razón por la cual es conveniente profundizar los estanques a 1,50 m., para evitar que los descensos de temperaturas excedan $\operatorname{los} 10^{\circ} \mathrm{C}$ en los sectores más profundos de los viveros donde se protegen los peces. Las temperaturas del agua a la que se adapta mejor el Piaractus Mesopotamicus (pacú) se sitúa por encima de los $22{ }^{\circ} \mathrm{C}$, ya que son peces de climas tropicales. La temperatura considerada óptima es entre 22 y $28{ }^{\circ} \mathrm{C}$, a menos de 15 ${ }^{\circ} \mathrm{C}$ los peces pierden el apetito y se reduce su crecimiento. A menos de $10{ }^{\circ} \mathrm{C}$ corren serios 
riesgos de supervivencia. El pacú necesita para lograr un buen crecimiento una concentración superior a $4 \mathrm{mg} / \mathrm{L}$ (miligramo de oxígeno por litro de agua). La incorporación de oxígeno al agua puede ser natural o artificialmente a través del aire de la atmósfera o la fotosíntesis. La zona de concentración letal es de 0 a $3 \mathrm{mg} / \mathrm{L}$ para el pacú. (Pacic 2010).

La piscicultura experimenta actualmente un creciente desarrollo en el NEA, pero adolece aún de algunos problemas que dificultan una mayor expansión. Dentro de los problemas a resolver y que atentan contra el desarrollo de la actividad, se encuentra el estancamiento del crecimiento de los peces durante el invierno debido a las bajas temperaturas. Cuando la temperatura del agua desciende por debajo de los $20^{\circ} \mathrm{C}$, los peces dejan de alimentarse, acotando el período de cultivo a 200-250 días dependiendo de las condiciones locales. A ello debe sumarse que en situaciones de fríos extremos y prolongados, la temperatura del agua del estanque puede descender a menos de $10^{\circ} \mathrm{C}$, favoreciendo la aparición de enfermedades e inclusive ocasionando la muerte de los peces.

El uso térmico de la energía solar para sistemas de calentamiento de agua es una de las tecnologías más difundidas a nivel mundial. Los invernaderos son los sistemas más aplicados a los estanques para piscicultura ya que proveen incrementos significativos en la temperatura del agua, sin embargo exhiben algunos inconvenientes como ser su alto costo inicial y complicada operación para evitar sobrecalentamientos durante el verano. Las otras alternativas para calentar el agua, como las bombas de calor, calderas a combustibles fósiles o resistencias eléctricas implican altos costos iniciales y operacionales y además tienen un impacto negativo para el ambiente a causa de la emisión de gases con efecto invernadero. Una medida adicional, en el caso de presentarse temperaturas bajas, es cubrir los estanques con mantas térmicas como las utilizadas en las piscinas. El problema que presenta esta alternativa es que reduce el intercambio de oxígeno con el aire, dificulta la manipulación de los peces y en general habría que ponerlo y quitarlo diariamente porque de lo contrario impediría el calentamiento diurno del estanque con la radiación solar. Otra opción es considerar calentar el agua por medio de captadores solares para temperaturas cercanas al ambiente, sin cubierta de vidrio, que pueden contribuir al incremento de la temperatura del agua hasta cerca de $5^{\circ} \mathrm{C}$ por sobre el ambiente. Otros inconvenientes asociados a esta tecnología son la necesidad de usar bombas para hacer circular el agua por los captadores con el consiguiente consumo de electricidad, el ensuciamiento que se podría producir debido a la elevada cantidad de sólidos disueltos que puede contener el agua del estanque y también elevados costos iniciales debido a las grandes superficies necesarias. Aparece como una alternativa a evaluar calentar "in situ" directamente el agua del estanque mediante los captadores solares flotantes y usar la gran masa del agua del estanque para almacenar energía y evitar descensos pronunciados de temperatura durante los días muy fríos, reduciendo simultáneamente la evaporación y las pérdidas por radiación nocturna hacia el cielo. (Farias et al., 2015).

Hasta el presente, numerosos autores han informado sobre el modelado térmico de un estanque de invernadero de piscicultura para predecir la temperatura del estanque durante todo el año. Klemestson y Rogers (1985) probaron un estanque de refugio de invernadero o plástico que podría lograr un aumento de $2,8-4,4^{\circ} \mathrm{C}$ en la temperatura del agua de cada mes del año en comparación con un estanque al aire libre.

Zhu et al., (1998) mediante modelizaciones analizaron las principales pérdidas de calor del agua para un sistema de invernadero y concluyeron que la mejor manera de reducirlas es controlando la convección entre la cubierta transparente y el aire exterior, la radiación térmica entre la cubierta transparente y el cielo y la radiación térmica entre la cubierta y la 
superficie del agua. Además reportaron que un sistema de estanques de invernadero puede lograr una mejora de $5,2{ }^{\circ} \mathrm{C}$ en la temperatura del agua de un estanque de $1 \mathrm{~m}$ de profundidad, en comparación con la temperatura del aire exterior.

Dilip (2007) presentó un modelo analítico transitorio de la eficiencia de un invernadero para calentamiento de un estanque para piscicultura. Los estudios paramétricos involucraron los efectos de longitud, ancho, profundidad e inclinación del revestimiento del estanque de peces. El rendimiento del estanque de peces se evaluó en términos de ganancia de temperatura, eficiencia térmica media y nivelación de la carga térmica. La principal restricción económica en las actividades invernales de un estanque de cultivo de peces es la necesidad de realizar operaciones en un estanque cubierto para mantener las temperaturas adecuadas del agua.

Se analizaron alternativas de bajo costo para el calentamiento de agua de estanques para piscicultura clasificados como semi intensivos a intensivos, según la capacidad de producción de 20 ton/Ha a 200 ton/ha. (Manual básico de Piscicultura en estanques, FAO 2010). Se presentan las evoluciones en función del tiempo para diferentes condiciones climáticas de: i) las temperaturas, ii) los flujos de calor intercambiados; para dos estanques: a) un estanque abierto a la atmósfera, utilizado como testigo, sin utilizar ningún dispositivo adicional y b) otro estanque en el que sucesivamente se ubicaron captadores flotantes experimentales de diferentes tipos para incrementar la temperatura. Se establece cuales son las características que más influyen y cuál es el dispositivo que mejor resultado ofrece.

\section{Materiales y Métodos}

Para ensayar los diferentes modelos de captadores solares se construyeron dos piletas, que simulan a un estanque a escala, de $0,65 \mathrm{~m}$ de profundidad, 0,60 $\mathrm{m}$ de ancho $\mathrm{x} 1,10 \mathrm{~m}$ de largo. Se utilizaron placas de $3 \mathrm{~cm}$ de espesor de poliestireno expandido como aislante en el fondo y las paredes. La impermeabilización se logró con un film de polietileno de color negro y la estructura se basó en un marco de perfiles de hierro, cerrado por chapas galvanizadas. La superficial de la interfase agua-aire es de $0,57 \mathrm{~m} 2$ por pileta, (Figura 1 ).

Para la determinación de la temperatura del agua en las piletas se realizaron mediciones cada 15 minutos mediante sensores de temperatura tipo Pt100 envainados en acero inoxidable, conectados mediante 3 conductores con un sistema de adquisición de datos de 6,5 dígitos de la marca Rigol. En cuanto a la medición de la temperatura ambiente, humedad relativa, velocidad del viento, radiación solar y otras variables meteorológicas se realizaron mediciones cada 15 minutos mediante una estación meteorológica modelo Vantage PRO2 de la marca Davis que incluye: pluviómetro, anemómetro y sensores de temperatura, humedad y radiación solar. Para mejor precisión, los sensores de humedad y temperatura estuvieron ubicados en su correspondiente abrigo meteorológico. 


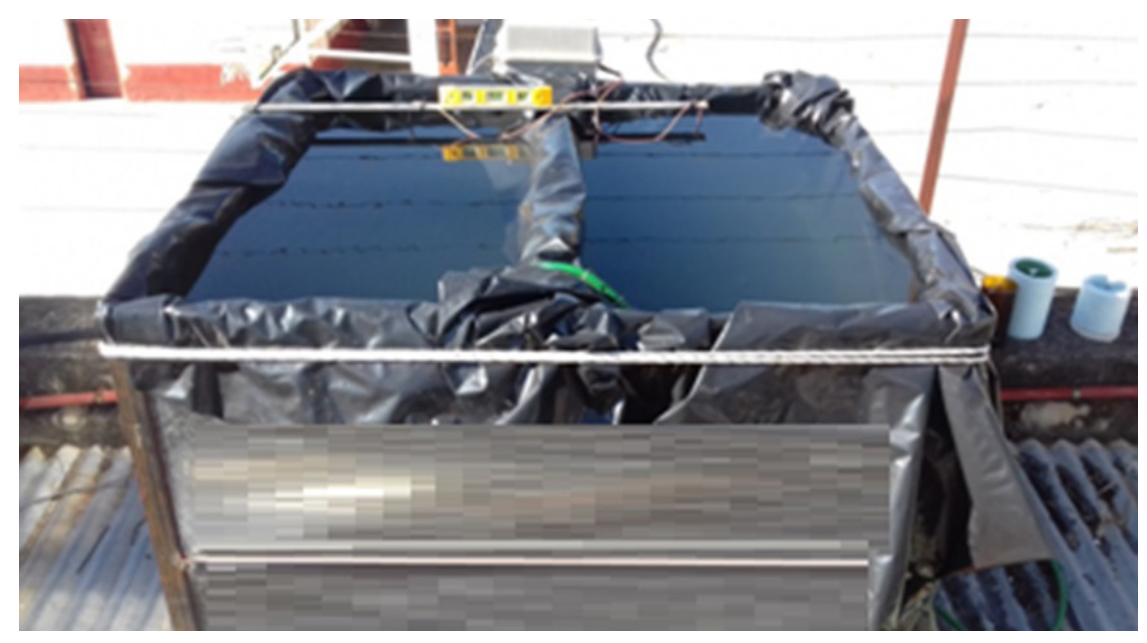

Figura 1: Piletas de Ensayo: Pileta 1 (izquierda) y Pileta 2 (derecha).

Atendiendo a las premisas que se habían establecido en la etapa de diagnóstico, entre ellas que el captador sea liviano, de bajo costo, construcción sencilla, sin partes en movimiento y sin consumo de energía, se diseñó en primer lugar un captador consistente en un marco rectangular de poliestireno expandido cubierto por una lámina de acetato, para favorecer el efecto invernadero y reducir las pérdidas por evaporación entre otras ventajas. Durante los ensayos se detectaron dos inconvenientes importantes: en primer lugar la cara inferior de la lámina de acetato se cubría de gotas debido a la condensación del vapor de agua, lo que disminuía la trasmitancia de radiación de la cubierta. En segundo lugar, sobre la superficie superior se acumulaba el agua del rocío nocturno y de lluvia. Para solucionar el primer inconveniente se ensayaron colectores de doble cubierta de acetato, la inferior en contacto directo con el agua y la superior en contacto con la atmósfera, generando en su interior una cámara estanca que evita la presencia de vapor y su posterior condensación. Estos colectores de doble cubierta se ensayaron y se obtuvieron mejoras térmicas importantes, pero adolecían todavía del inconveniente del agua de rocío y de lluvia que a su vez favorecían el rápido y progresivo ensuciamiento de la cubierta superior.

Para solventar este último inconveniente se diseñó un captador con la superficie superior con una doble pendiente en $\mathrm{V}$ invertida, de forma que se escurra el agua de rocío y de lluvia, favoreciendo su secado y reduciendo consecuentemente el ensuciamiento.

El captador está construido en base a tres mamparos de poliestireno expandido de alta densidad, que le dan forma y estructura al estar recubiertos por una sola lámina de acetato que hace de cubierta inferior, rodea a los mamparos y también hace de cubierta superior con pendiente " a dos aguas" (Figura 2). Entre las dos caras y los mamparos se forman 2 volúmenes estancos que le provén flotabilidad, generan el efecto invernadero y minimizan las pérdidas de calor por convección y evaporación del agua. 

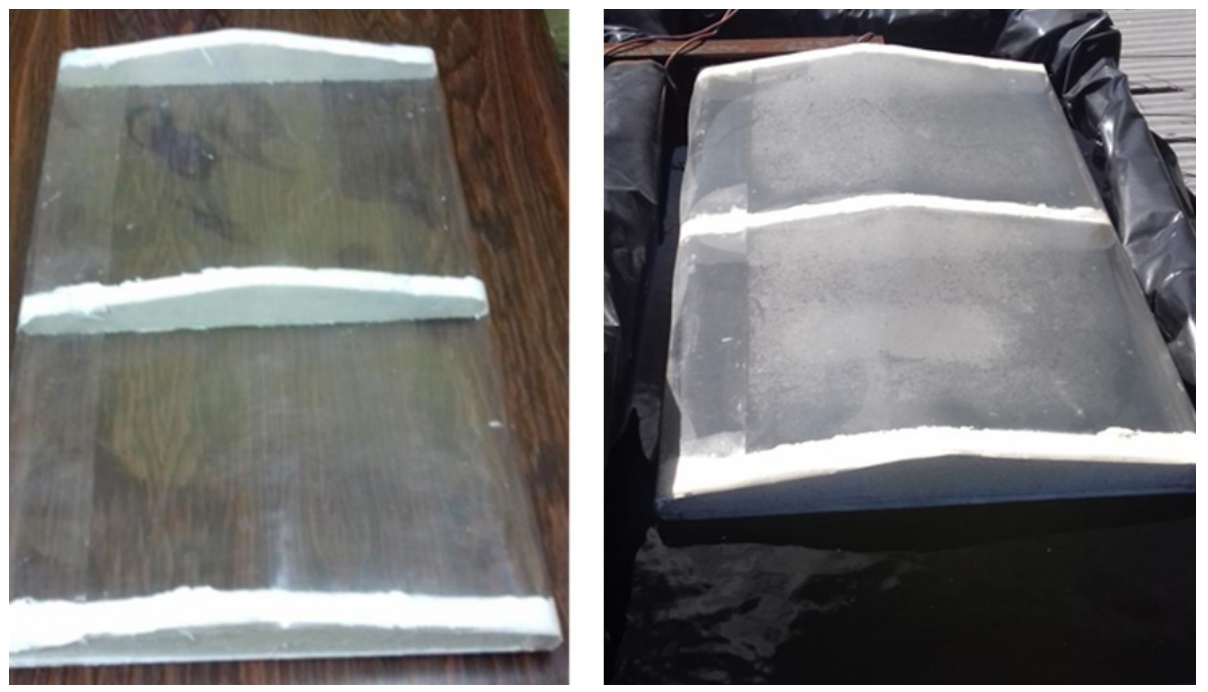

Figura 2: Vistas del captador flotante curvo

\section{Balance energético}

En un trabajo previo se desarrolló un modelo termodinámico simple que describe los cambios en la energía interna de las piletas al aire libre que simulan los estanques de acuicultura, (Gutierres et al., 2018). Suponiendo despreciables los flujos de energía relacionados con las filtraciones de agua hacia las napas subterráneas, los originados en las lluvias, en los desbordamientos de agua, los relacionados al agua de reposición y a los intercambios con las paredes de las piletas, la conservación de la energía en estado transitorio estará dada por:

$$
\left(\frac{d U}{d t}\right)=q_{\text {rad }}-q_{\text {evap }} \pm q_{\text {fondo }} \pm q_{c o n v}
$$

Ver apéndice de nomenclaturas.

La energía interna dentro del volumen de control está dado por:

$$
U=m_{w} C T
$$

En el modelo la masa de agua se considera constante; también se asume que la densidad y el calor específico se mantienen constantes con la temperatura, ya que las variaciones de estas con cambios en la temperatura son despreciables. El calor neto intercambiado incluye el calor intercambiado por radiación, por conducción a través del fondo, por convección en la superficie y el necesario aporte para la evaporación del agua.

$$
Q_{\text {neto }}=q_{\text {rad }}-q_{\text {evap }} \pm q_{\text {fondo }} \pm q_{\text {conv }}
$$

Durante las experiencias se mide el tiempo y la temperatura del agua de las piletas, por lo cual se puede calcular la derivada de la temperatura con respecto al tiempo. 


$$
\left(\frac{d T}{d t}\right)=\frac{Q_{n e t o}}{m_{w} C}
$$

Conocidos la masa del agua contenida en la pileta y la derivada de la temperatura con respecto al tiempo se puede calcular el calor neto intercambiado.

$$
Q_{\text {neto }}=m_{w} C\left(\frac{d T}{d t}\right)
$$

Simultáneamente se mide la radiación solar incidente sobre la superficie libre del agua de la pileta. Entonces se podría obtener el flujo de calor combinando conducción, convección y evaporación.

$$
Q_{\text {neto }}-q_{\text {rad }}= \pm q_{\text {fondo }} \pm q_{\text {conv }}-q_{\text {evap }}
$$

\section{RESULTADOS}

\section{Experiencias con captador flotante curvo}

En la figura 3 se exponen parte de los resultados de una experiencia realizada desde el 20/06/19 a las 12:00 pm hasta el día 16/08/19 a las 10:20 am. El periodo seleccionado corresponde a un periodo de tiempo frío y estable, desde el 14/07 a las 00:00 am hasta el día 19/07 a las 23:00 pm, con temperatura ambiente máxima de $24,2{ }^{\circ} \mathrm{C}$ y mínima de $8,4{ }^{\circ} \mathrm{C}$, el día $19 / 07$ se produjo una débil lluvia entre las 01:30am y las 02:30 am.

En los gráficos, la temperatura del agua de la pileta con el captador se designa como T_c/ col, y corresponde a la línea continua de color negro, mientras que la temperatura del agua de la pileta testigo se nombra como T_s/col y corresponde a la línea continua de color rojo. La temperatura del aire ambiente (T_amb) se representa con la línea color verde. Todas las temperaturas están expresadas en grado Celsius. La velocidad del viento promedio para un periodo de 15 minutos (Vel.V) está en $\mathrm{Km} / \mathrm{h}$. Para el día 14/07 la velocidad del viento fue baja, y los demás días no tuvieron un valor significativo. A pesar de esto, las curvas de calentamiento y enfriamiento mantienen su comportamiento, por lo cual podría decirse que éste fenómeno no influye en las pendientes. La humedad que se representa en los gráficos se refiere a la humedad relativa, la cual tuvo un valor promedio del $75 \%$ para los días de ensayo. La radiación solar sobre superficie horizontal máxima alcanzada fue de $583 \mathrm{~W} / \mathrm{m} 2$, a las 13:00hs el día 17/07, presentando los días cercanos radiaciones máximas similares.

En la pileta 2 se emplazó el captador curvo. La superficie ocupada por el captador en la pileta de ensayo es de 0,32 m2, lo cual representa un $56 \%$ de la superficie total, (donde de ese $\%$ un $87 \%$ corresponde a la parte transparente y el 13\% restante corresponde a la superficie de los bordes), el porcentaje restante un $44 \%$ es la superficie no cubierta en la pileta, se deja con el fin de favorecer la oxigenación del agua.

En el gráfico se visualiza una mayor pendiente en las curvas de calentamiento y enfriamiento para la temperatura en la pileta en la que no se instaló el captador (línea continua 
roja). Lo cual significa que este proceso ocurre más rápido que la temperatura del agua de la pileta que posee el captador. Cuando la temperatura del aire exterior alcanza los valores más bajos el cambio es menos pronunciado en la pileta que posee en captador y es más pronunciado en la pileta sin el mismo.

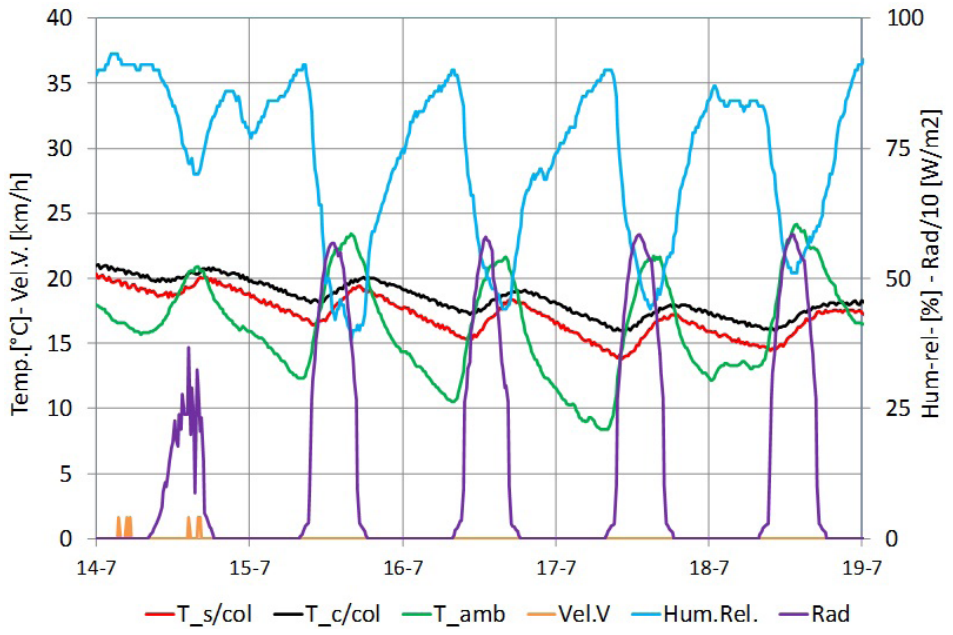

Figura 3: Temperatura de las piletas, ambiente, velocidad del viento, humedad y radiación para 5 días de tiempo estable entre el 14 y el 19 de julio.

En la figura 4 se exponen parte de los resultados de una experiencia realizada desde el 20/06/19 a las 12:00 pm hasta el día 16/08/19 a las 10:20 am. El periodo seleccionado corresponde a un periodo de tiempo frío y estable, desde el 02/08 a las 00:00 am hasta el día 06/08 a las $23: 00 \mathrm{pm}$, con temperatura ambiente máxima de $23,3{ }^{\circ} \mathrm{C}$ el día $05 / 08$ a las $15: 45 \mathrm{hs}$ y mínima de $6,7^{\circ} \mathrm{C}$ el día $03 / 08$ a las $07: 45$ hs.

Para los días $02 / 08$ y 03/08 la velocidad del viento fue muy baja (menor que $0,5 \mathrm{~m} / \mathrm{s}$ ) y los demás días no tuvieron un valor significativo. La humedad relativa tuvo un valor promedio del $64 \%$ para los días de ensayo. La radiación solar sobre superficie horizontal máxima alcanzada fue de $662 \mathrm{~W} / \mathrm{m} 2$ el día 04/08, a las 13:00 hs presentando los días cercanos radiaciones máximas similares. Los valores máximos de temperatura del aire se alcanzan en horas desde las 15:30 y las 16:00 hs.

Al igual que en la experiencia anterior, en la pileta 2 se emplazó el captador curvo y en el gráfico se visualiza una mayor pendiente en las curvas de calentamiento y enfriamiento para la temperatura en la pileta en la que no se instaló el captador. Lo cual significa que este proceso ocurre más rápido que la temperatura del agua de la pileta que posee el captador. 


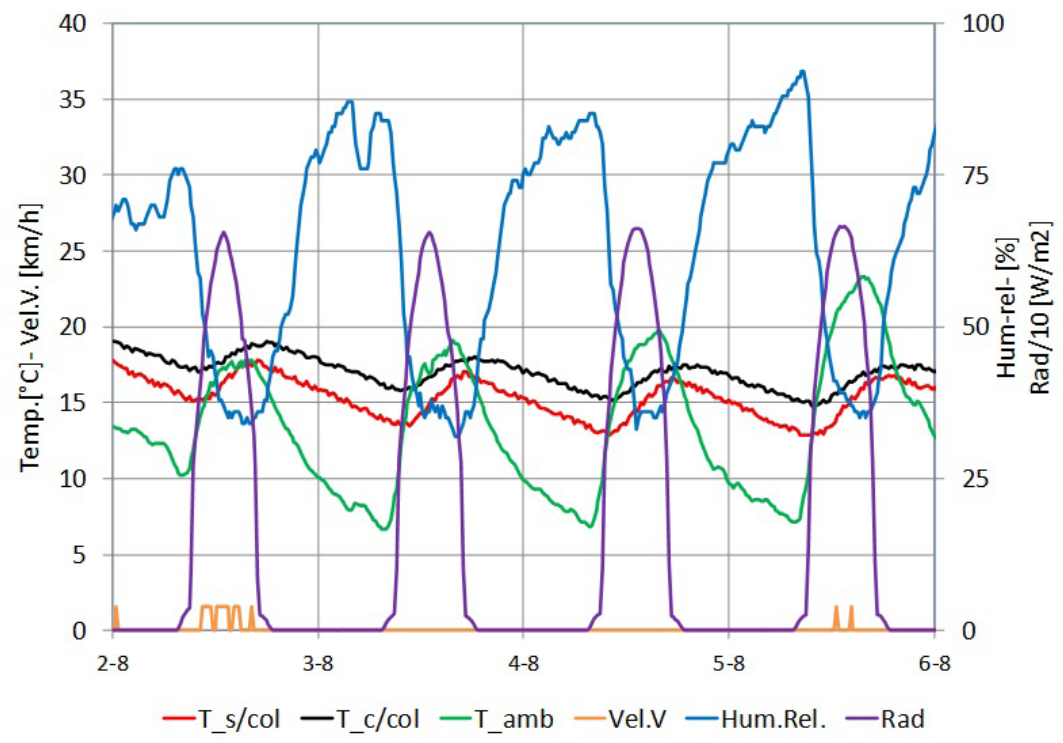

Figura 4: Temperatura de las piletas, ambiente, velocidad del viento, humedad y radiación para 4 días de tiempo estable entre el 02 y el 06 de agosto.

Observando las figuras 3 y 4 , se visualiza que hay cuatro días totalmente despejados y calmos de características similares (del 15/7 al 18/7 y del 02/8 al 05/8), entre los cuales se compara los parámetros climáticos, presentando en el cuadro siguiente los valores promedios:

\begin{tabular}{|c|c|c|}
\hline Variable & Promedio 15-18/07 & Promedio 02-05/08 \\
\hline Radiación Máxima [W/m2] & 575 & 656 \\
\hline Irradiación diaria [M]/m2] & 11,6 & 14,5 \\
\hline T_amb_min $\left[{ }^{\circ} \mathrm{C}\right]$ & 10,9 & 7,7 \\
\hline T_agua_s/col_min $\left[{ }^{\circ} \mathrm{C}\right]$ & 15,4 & 13,6 \\
\hline T_agua_c/col_min $\left[{ }^{\circ} \mathrm{C}\right]$ & 17,1 & 15,7 \\
\hline T_amb_med $\left[{ }^{\circ} \mathrm{C}\right]$ & 16 & 13,4 \\
\hline T_agua_s/col_med $\left[{ }^{\circ} \mathrm{C}\right]$ & 16,6 & 15,3 \\
\hline T_agua_c/col_med $\left[{ }^{\circ} \mathrm{C}\right]$ & 18 & 17 \\
\hline T_agua_s/col_min - T_amb_min $\left[{ }^{\circ} \mathrm{C}\right]$ & 4,5 & 5,9 \\
\hline T_agua_c/col_min - T_amb_min $\left[{ }^{\circ} \mathrm{C}\right]$ & 6,2 & 8,0 \\
\hline T_agua_c/col_min - T_agua_s/col_min $\left[{ }^{\circ} \mathrm{C}\right]$ & 1,7 & 2,1 \\
\hline
\end{tabular}

Tabla 1: Comparación de radiación solar e irradiación diaria para 4 días del mes de julio y 4 días del mes de agosto. Evaluación de la incidencia en las temperaturas ambiente y del agua.

Se observa que los días del mes de agosto bajo estudio fueron días más fríos que los del mes de julio, la temperatura mínima promedio para los días de agosto fue de $7,7{ }^{\circ} \mathrm{C}$ mientras que para los días de julio fue de $10,9^{\circ} \mathrm{C}$. Sin embargo, a pesar de ello, según los valores 
calculados, la diferencia de la temperatura alcanzada entre el agua de las piletas y la ambiente fue mayor para el mes de agosto. Además, también se observa una mayor de diferencia entre el agua de las piletas para el mes de agosto. En estas diferencias puede considerarse del impacto de la influencia de los valores de radiación solar y la irradiación diaria entre los meses considerados, la cual fue más alta para dicho mes.

En la figura 5 se exponen parte de los resultados de una experiencia realizada desde el desde el 20/06/19 a las 12:00 pm hasta el día 16/08/19 a las 10:20 am. El periodo seleccionado, desde 22/07 a las 00:00 am hasta el día 28/07 a las 23:00 pm, corresponde a un pronunciado descenso de la temperatura ambiente desde una máxima de $27,3^{\circ} \mathrm{C}$ antes de la ola de frío hasta una mínima de $7,7^{\circ} \mathrm{C}$ el día $26 / 07$ a las 07:00 am. El día 23/07 se produjo una débil lluvia a las 06:00 am y también llovió entre las 13:30 pm y las 14:45 pm del mismo día. El día 24/07 el viento sopló durante todo el día, alcanzando la máxima de $6,4 \mathrm{~km} / \mathrm{h}$ a las 09:45 am, teniendo los demás días velocidades menores. La humedad relativa tuvo un valor promedio del 81 $\%$ para los días de ensayo. La radiación solar máxima alcanzada fue de $536 \mathrm{~W} / \mathrm{m} 2$, a las 13:00 hs el día 22/07 y posteriormente se sucedieron varios días nublados con escasa radiación.

En la pileta 2 se emplazó el captador curvo. Para el día más frío, con una temperatura ambiente mínima de $7,7{ }^{\circ} \mathrm{C}$ la temperatura mínima del agua de la pileta con el captador fue de $11,7^{\circ} \mathrm{C}$ es decir $4{ }^{\circ} \mathrm{C}$ por sobre la ambiente mientras que para la pileta testigo fue de 2,2 ${ }^{\circ} \mathrm{C}$ sobre el ambiente. La máxima diferencias de temperatura de la pileta con el captaldor y la pileta testigo es aproximadamente de $2{ }^{\circ} \mathrm{C}$ para horarios alrededor de las 10 y las $11 \mathrm{am}$.

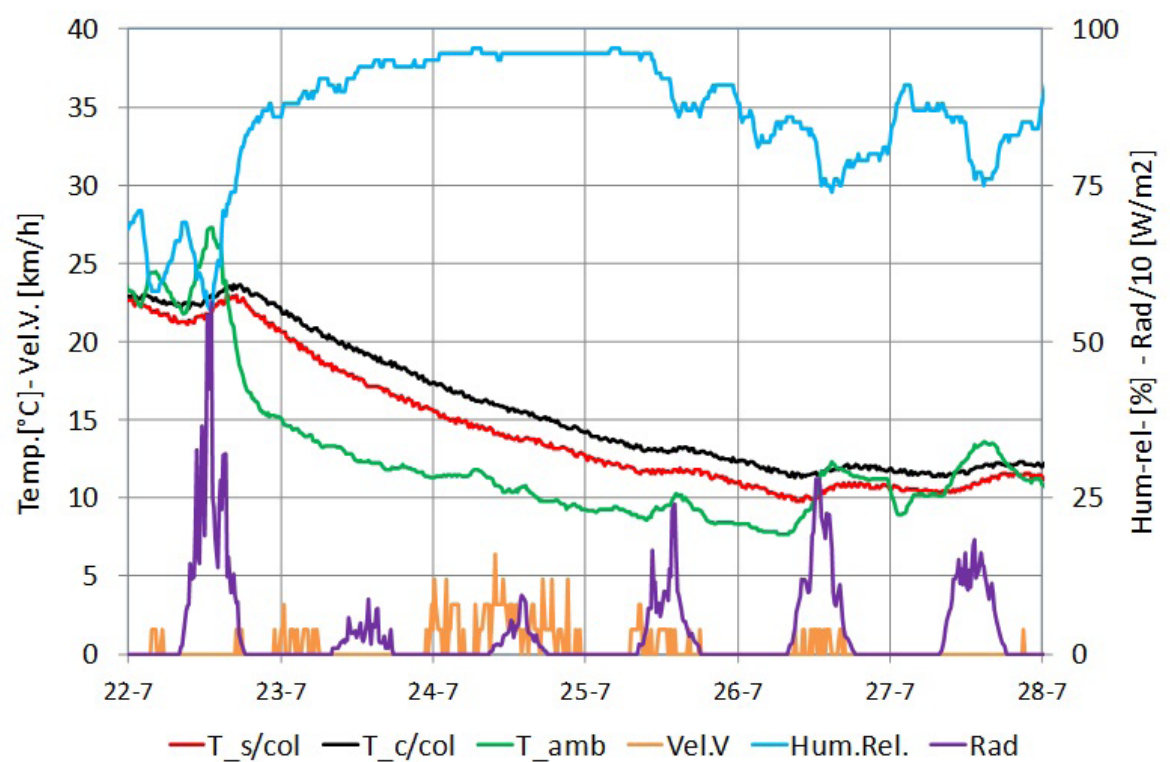

Figura 5: Temperatura de las piletas, ambiente, velocidad del viento, humedad y radiación para 6 días de tiempo estable entre el 22 y el 28 de julio.

Para efectuar un análisis energético, a partir de los datos experimentales, se determinaron las pendientes promedio de las curvas de temperatura en función del tiempo para los periodos estables de calentamiento (entre las 12 y $16 \mathrm{hs}$ ) y los de enfriamiento (entre las 19 y $6 \mathrm{hs}$ ), dichos resultados se exponen en la tabla 2. A partir de estas pendientes se determinaron, mediante la ecuación (5), los flujos netos de calor intercambiados entre el agua de 
las piletas y el ambiente, considerando que la masa de agua contenida en las piletas es de $320 \mathrm{~kg}$.

\begin{tabular}{|c|c|c|c|c|c|c|c|c|}
\hline \multirow{2}{*}{ Pileta } & \multirow{2}{*}{ Proceso } & \multicolumn{5}{|c|}{ Pendientes de la evolución de las temperaturas [K/día] } & \multirow{2}{*}{$\frac{\text { Prom. }}{\text { [K/día] }}$} & \multirow{2}{*}{$\begin{array}{l}\text { Flujo Neto } \\
\text { de calor [W] }\end{array}$} \\
\hline & & $14 / 07$ & $15 / 07$ & $16 / 07$ & $17 / 07$ & $18 / 07$ & & \\
\hline \multirow{2}{*}{$\mathrm{c} / \mathrm{col}$} & Cal. & 2,8 & 8,0 & 6,5 & 6,8 & 7,4 & 7,2 & 108 \\
\hline & Enf. & $-4,1$ & $-4,0$ & $-4,9$ & $-3,2$ & $-0,2$ & $-3,1$ & -46 \\
\hline \multirow{2}{*}{ s/col } & Cal. & 8,0 & 13,9 & 14,1 & 14,1 & 11,0 & 13,3 & 212 \\
\hline & Enf. & $-4,4$ & $-6,2$ & $-6,7$ & $-4,6$ & $-0,8$ & $-4,6$ & -73 \\
\hline
\end{tabular}

Tabla 2: Pendientes de las evoluciones de temperaturas y flujos netos de calor hacia piletas para los periodos de calentamiento (12:00 a 16:00hs) y de enfriamiento (19:00 hs a 6:00 am).

El flujo neto de calor determinado corresponde al indicado en la ecuación (7) y tiene en cuenta la sumatoria de los flujos que entran y salen del sistema bajo análisis. Según el cuadro se obtuvieron resultados experimentales que indican las magnitudes para dos condiciones: "cal" desde las 12:00 hasta las 16:00hs y "enf" desde las 19:00 hs a 6:00 am. Comparando los resultados obtenidos para los dos casos, se observa que tanto la ganancia como la pérdida de calor al exterior ocurren más rápidamente en el caso de la pileta sin captador que en la pileta con el captador. El Flujo Neto de calor durante las horas de enfriamiento para la pileta sin el captador es de $-73 \mathrm{~W}$, mientras que para la pileta con el captador es de $-46 \mathrm{~W}$. El Flujo Neto de calor durante las horas de calentamiento para la pileta con el captador es de $108 \mathrm{~W}$, mientras que para la pileta sin el captador es de $212 \mathrm{~W}$.

La radiación solar en $\mathrm{W} / \mathrm{m}^{2}$ se obtuvo mediante la estación meteorológica, sabiendo que el área de las piletas es $0,57 \mathrm{~m}^{2}$ se obtiene la potencia total que incide sobre cada pileta en Watts durante el periodo de calentamiento, expuestos en la tabla 3.

\begin{tabular}{|c|c|c|c|c|c|c|}
\hline Variable & $14 / 07$ & $15 / 07$ & $16 / 07$ & $17 / 07$ & $18 / 07$ & Promedio \\
\hline Radiación [W/m2] & 236 & 518 & 486 & 534 & 534 & 518 \\
\hline Potencia [W] & 134 & 295 & 277 & 304 & 304 & 295 \\
\hline
\end{tabular}

Tabla 3: Radiación promedio incidente en las piletas para horarios de calentamiento (12:00 a 16:00hs)

La potencia promedio incidente sobre cada una de las piletas para los días ensayados es de $295 \mathrm{~W}$ durante las horas de calentamiento. Conociendo el flujo neto de calor y la radiación solar incidente se pueden determinar los flujos de calor restantes, agrupados según la ecuación (6). La diferencia entre la potencia y el flujo neto de calor durante las horas de calentamiento para la pileta sin captador es de $83 \mathrm{~W}$, mientras que para la pileta con el captador es de 187 W, ambos flujos integran los fenómenos de convección, evaporación y conducción.

En la tabla 4 y la tabla 5 se exponen valores medios diarios de las temperaturas relevantes. Durante las horas de calentamiento (12:00 a 16:00 hs), el promedio de la temperatura de agua de la pileta s/col fue de $17,2{ }^{\circ} \mathrm{C}$ (para 5 días de ensayo), para $18,3{ }^{\circ} \mathrm{C}$ para la pileta $\mathrm{c} / \mathrm{col} \mathrm{y}$ 
la temperatura del aire de $21,2^{\circ} \mathrm{C}$. Durante las horas de enfriamiento (19:00 hs a 6:00 am), el promedio de la temperatura de agua de la pileta s/col fue de $17,2^{\circ} \mathrm{C}$ (para 5 días de ensayo), para $18,4{ }^{\circ} \mathrm{C}$ para la pileta c/col y la temperatura del aire de $14,4{ }^{\circ} \mathrm{C}$.

\begin{tabular}{|c|c|c|c|c|c|c|}
\hline Variable & $14 / 07$ & $15 / 07$ & $16 / 07$ & $17 / 07$ & $18 / 07$ & Promedio \\
\hline T_s/col & 19,19 & 18,1 & 17,0 & 15,7 & 16,2 & 17,2 \\
\hline T_c/col & 20,2 & 19,2 & 18,2 & 17,0 & 17,1 & 18,3 \\
\hline T_amb & 19,7 & 22,1 & 20,6 & 20,5 & 23,3 & 21,2 \\
\hline T_c/col -T_s/col & 1,0 & 1,0 & 1,1 & 1,3 & 1,0 & 1,1 \\
\hline T_amb - T_c/col & $-0,5$ & 2,9 & 2,4 & 3,6 & 6,2 & 2,9 \\
\hline
\end{tabular}

Tabla 4: Temperaturas promedio del agua de las piletas, del ambiente y sus diferencias, durante el proceso diario de calentamiento (12:00 a 16:00hs)

El aumento de la temperatura del agua entre la pileta con el captador solar y sin el mismo fue de $1,1^{\circ} \mathrm{C}$ en promedio (para los 5 días ensayados) durante las horas de calentamiento y de $1,3{ }^{\circ} \mathrm{C}$ para las horas de enfriamiento. Se observa que para las horas de calentamiento la temperatura del aire fue de $2,9^{\circ} \mathrm{C}$ (promedio) superior que el agua de la pileta con captador, mientras que para las horas de enfriamiento la temperatura del agua fue $4{ }^{\circ} \mathrm{C}$ superior que la temperatura del aire, lo cual es favorable ya que para éste período es necesario mantener la temperatura del agua para que se alcancen temperaturas que ronden los $20-24{ }^{\circ} \mathrm{C}$ en los meses de invierno.

\begin{tabular}{|c|c|c|c|c|c|c|}
\hline Variable & $14 / 07$ & $15 / 07$ & $16 / 07$ & $17 / 07$ & $18 / 07$ & Promedio \\
\hline T_s/col & 18,6 & 17,6 & 16,5 & 15,9 & 17,4 & 17,2 \\
\hline T_c/col & 19,8 & 19,1 & 17,9 & 17,2 & 18,1 & 18,4 \\
\hline T_amb & 15,6 & 14,5 & 11,6 & 13,5 & 17,0 & 14,4 \\
\hline T_c/col -T_s/col & 1,2 & 1,5 & 1,5 & 1,3 & 0,7 & 1,3 \\
\hline T_amb - T_c/col & $-4,2$ & $-4,6$ & $-6,4$ & $-3,7$ & $-1,1$ & $-4,0$ \\
\hline
\end{tabular}

Tabla 5: Temperaturas promedio del agua de las piletas, del ambiente y sus diferencias, durante el proceso diario de enfriamiento (19:00 a 6:00hs)

\section{Discusión de resultados}

Zhu et al., (1998) simularon mediante un programa informático el comportamiento de un invernadero con un recubrimiento de polietileno durante los meses de invierno, en Bélgica, obteniéndose que la variación de temperatura del agua de un estanque en promedio pueden aumentar en $5,2^{\circ} \mathrm{C}$ en comparación con la temperatura del aire ambiente exterior. Las experiencias con los captadores solares flotantes demuestran que se pueden obtener efectos similares. Según la tabla 1, las diferencias de temperaturas, considerando los valores medios, entre la temperatura del agua con el captador y la exterior fueron de $2{ }^{\circ} \mathrm{C}$, para 4 días de julio y $3,6^{\circ} \mathrm{C}$ para 4 días del mes de agosto. En las tablas 4 y 5 , se muestra que considerando 
las temperaturas promedio del agua y del aire para los períodos de calentamiento y enfriamiento, la diferencia entre la temperatura del agua y del aire se encuentra entre 3 y 4 ${ }^{\circ} \mathrm{C}$. Aplicando el modelo, Zhu et al., (1998) determinaron las temperaturas del aire en el interior del invernadero y la temperatura del agua para un invernadero de polietileno para los meses de invierno. Para el caso extremo de temperaturas mínimas se extrae el dato de que la diferencia de temperatura entre el agua y la temperatura del aire exterior es de $10^{\circ} \mathrm{C}$. Comparando este dato con las figuras 3 y la figura 4, se observa que para los días donde se alcanzan las temperaturas ambientes mínimas, la diferencia de temperatura entre la del agua y la del ambiente es de $8^{\circ} \mathrm{C}$ (Figura 3 ) y de $9^{\circ} \mathrm{C}$ (figura 4), los cuales son valores cercanos a los obtenidos por Zhu et al., (1998). También se publicó la evaluación del calentamiento del estanque con invernaderos de PVC, vidrio y vidrio de baja emisividad, materiales que poseen rendimientos similares al polietileno alcanzando las diferencias de las temperaturas medias entre el aire externo y el agua alrededor de $5{ }^{\circ} \mathrm{C}$, presentando el vidrio menor rendimiento. Se debe mencionar también que durante los ensayos la temperatura ambiente supera a la temperatura del agua desde horarios cercanos a las $11 \mathrm{hs}$, hasta las $17 \mathrm{hs}$ aproximadamente donde decrece y se encuentra por debajo del valor de la temperatura de las piletas. En el modelo del invernadero utilizado como comparación, la temperatura del agua siempre está por encima del valor de la temperatura del aire exterior. Dilip J. (2007) desarrolló un modelo térmico de un estanque para piscicultura con un invernadero y obtuvo que la temperatura mínima del agua a la mañana temprano desciende hasta $16{ }^{\circ} \mathrm{C}$ cuando la temperatura externa del aire ambiente es de $5{ }^{\circ} \mathrm{C}$, es decir una diferencia entre las temperaturas mínimas de $11{ }^{\circ} \mathrm{C}$, en contraste, utilizando los captadores curvos se obtuvo una diferencia de hasta 9 ${ }^{\circ} \mathrm{C}$, valor menor originado en que solo se cubre un $44 \%$ de la superficie del estanque con un sistema más sencillo y económico. Por otra parte Sarkar and Tiwari (2006) en un estudio similar de simulación de un estanque ubicado a una latitud de $29^{\circ} \mathrm{N}$ en la región del Himalaya obtuvo que en promedio el invernadero puede aumentar en $4,14{ }^{\circ} \mathrm{C}$ la temperatura del agua de un estanque con invernadero en relación a otro expuesto al ambiente.

Una manta térmica flotante se utilizó para el aumentar la temperatura del agua de un estanque en Australia (Wisely et al., 1981). Se comparó la variación de la temperatura del agua con dos estanques de control: uno con renovación de agua y el otro sin renovación, utilizando los datos suministrados por una estación meteorológica. La manta consistía en dos láminas plásticas con burbujas llenas de aire, las cuales pueden aumentar la temperatura del agua de las piscinas entre $5^{\circ} \mathrm{C}$ y $8{ }^{\circ} \mathrm{C}$ (según reportes de los piscicultores). Los resultados de esta experiencia demostraron que la temperatura en el estanque cubierto aumenta rápidamente durante los primeros 7 días y después mantiene niveles diarios promediando $29^{\circ} \mathrm{C}$ (rango de 26 a $\left.31^{\circ} \mathrm{C}\right)$ a las $06: 00 \mathrm{hs} \mathrm{y} 32^{\circ} \mathrm{C}\left(29-34^{\circ} \mathrm{C}\right)$ a las 18:00hs. Las temperaturas mínimas y máximas promedio del agua en el estanque de control sin renovación se encuentran entre $22{ }^{\circ} \mathrm{C}$ y $26{ }^{\circ} \mathrm{C}$ respectivamente; y en el estanque de control con renovación de agua dichas temperaturas se encuentran entre $20{ }^{\circ} \mathrm{C}$ y $24^{\circ} \mathrm{C}$. La temperatura del aire ambiente promedia $14^{\circ} \mathrm{C}$ a las 6:00 hs y $19^{\circ} \mathrm{C}$ a las $18 \mathrm{hs}$. Las temperaturas media diaria del agua en el estanque cubierto fueron por ello de 7 a $9^{\circ} \mathrm{C}$ más altas que en los estanques de control temprano en la mañana y 6 a $7{ }^{\circ} \mathrm{C}$ en la tardecita. Después de 3 semanas la manta térmica se inutilizó debido a que se despegaron las capas de plástico debido a que la temperatura excedió los $30^{\circ} \mathrm{C}$. Teniendo en cuenta éstos valores se puede decir que el uso de las mantas es similar al efecto que tiene el uso de invernaderos, porque en ambos en todo momento la temperatura el agua del estanque está por encima de la temperatura ambiente. Para el caso de los captadores solares, la situación es algo diferente ya que en el horario comprendido entre las 10:00 hs y 
las 17:00 hs, aproximadamente, la temperatura del aire ambiente es mayor a la temperatura del agua. Fuera de ese periodo mencionado la temperatura del agua se encuentra por sobre la del ambiente. Cabe mencionar también que los captadores fabricados no cubren toda el área superficial de la pileta, quedando un $44 \%$ libre, por lo cual esto también incide en la diferencia del comportamiento con las mantas y los invernaderos en donde toda la superficie se encuentra protegida y aislada del aire exterior. Según Wisely et al., (1981) el uso de las mantas térmicas se vería dificultado en la región del NEA debido a que hay días calurosos, incluso durante los meses de invierno, donde se alcanzan temperaturas superiores a $30{ }^{\circ} \mathrm{C}$, lo que podría ocasionar una separación de las capas de la manta térmica ocasionando la destrucción de la misma en periodos de tiempo menores a un mes. También Gallardo et al., (2006) evaluaron el funcionamiento de las mantas térmicas para calefaccionar un estanque para cría de peces en Santiago de Cali, Colombia, ubicada a una latitud de $4{ }^{\circ} \mathrm{N}$, a una altitud de $1849 \mathrm{msnm}$, con precipitación anual de $1574 \mathrm{~mm}$, humedad relativa de alrededor del $85 \%$ y una temperatura promedio del aire de $17{ }^{\circ} \mathrm{C}$. Concluyeron que el uso de la manta térmica reduce las pérdidas de calor hasta en un $50 \%$ y son preferibles por su bajo costo, fácil adquisición y porque los colectores solares de placa plana presentan inconvenientes técnicos para su instalación y costos más elevados.

\section{Conclusiones}

Los ensayos realizados muestran que para la región del Nordeste Argentino con el uso de los captadores flotantes curvos se pueden conseguir incrementos de la temperatura mínima del agua de $6,2{ }^{\circ} \mathrm{C}$ durante julio y de $8{ }^{\circ} \mathrm{C}$ durante agosto en relación con la temperatura ambiente durante los horarios más críticos, temprano en la mañana. Simultáneamente, si se comparan ambos estanques (con y sin colector) se observa que las diferencias de temperaturas mínimas ascienden a $1,7{ }^{\circ} \mathrm{C}$ durante julio y $2,1{ }^{\circ} \mathrm{C}$ en agosto. También se observó que durante la tarde de días de tiempo estable la temperatura ambiente es superior a la temperatura de ambos estanques.

Durante una ola de frío del mes de julio, en la que hubo 6 días consecutivos muy nublados, para el día más frío, con una temperatura ambiente mínima de $7,7^{\circ} \mathrm{C}$ la temperatura mínima del agua de la pileta con el captador fue de $11,7^{\circ} \mathrm{C}$ es decir $4^{\circ} \mathrm{C}$ por sobre la del ambiente.

A partir de las pendientes de las curvas de temperatura-tiempo se estableció que el flujo neto de calor durante el periodo diario de calentamiento en promedio es de $108 \mathrm{~W}$ (ingresando), teniendo en cuenta que durante ese periodo se recibió una radiación solar de $295 \mathrm{~W}$ se concluye que se trasmitió por otros fenómenos (convección radiación, conducción) 187 W hacia el ambiente.

El captador flotante curvo mostró buen comportamiento antes las lluvias que no penetraron al interior y escurrieron rápidamente, también antes los vientos ya que a pesar de ser muy livianos no fueron arrastrados durante las tormentas y tampoco el ensuciamiento fue relevante luego de estar expuesto más de dos meses a la intemperie. 


\section{Apéndice: Nomenclatura}

\begin{tabular}{|c|c|l|}
\hline Magnitud & Unidad & \multicolumn{1}{|c|}{ Descripción } \\
\hline A & m2 & Área del estanque \\
\hline C & kJ/kg & Calor específico del agua \\
\hline U & J & Energía interna del estanque \\
\hline mw & Kg & Masa del agua \\
\hline qconv & W & Flujo de calor por convección. \\
\hline qevap & W & $\begin{array}{l}\text { Flujo de energía perdida a través de la evaporación del } \\
\text { agua. }\end{array}$ \\
\hline qrad & W & Flujo de calor por radiación \\
\hline qfondo & W & Flujo de calor por conducción por el fondo. \\
\hline Qneto & W & Flujo Neto de calor \\
\hline T & ${ }^{\circ} \mathrm{C}$ & Temperatura promedio del agua en el estanque. \\
\hline t & seg & Tiempo \\
\hline
\end{tabular}




\section{REFERENCIAS}

Dilip J. (2007). Modeling the thermal performance of an aquaculture pond heating with greenhouse. Building and environment 42, pp. 557-565.

Farías P., Sandoval M., Sanchez S., Figueredo G. Revisión de alternativas tecnológicas para el calentamiento solar de estanques para acuicultura (2015). Acta de la XXXVIII Reunión de Trabajo de la Asociación Argentina de Energías Renovables y Medio Ambiente. Vol. 3, pp. 02.21-02.32, 2015.

Gallardo Francisco J. Borja, González Saucedo Luis O.,. Quintero del Vallejo Victoria E. (2006). Evaluación de Alternativas para climatización de estanques para el cultivo de Tilapia Roja, localizados en la zona fría del Valle de Cauca, Colombia.

Gutierres Sosa, M. Y., Spotorno R., Pochettino J. J., Zurlo H. y Figueredo G. R. (2018). Modelización Termodinámica para estanques de Piscicultura. pp. 4-6.

Klemetson S.L., Rogers G.L. (1985) Aquaculture pond temperature modeling. Aquacult Eng; 4 pp.191-208.

Manual básico de Piscicultura en estanques. (2010). Dirección Nacional de Recursos Acuáticos. Uruguay. Montevideo, DINARA-FAO, 50 p.

Molina Martínez J.M., Martínez Álvarez V., Baille A. y González-real M.M. (2006).

Estimación de la evaporación en embalses de riego mediante un modelo de balance de energía. En: Ingeniería Del Agua Vol. 13 pp. 3.

Pacic Antonio (2010) Cría de Pacú en Cautiverio. INTA, Serie de Piscicultura 0001.

Consultado en: https://inta.gob.ar/sites/default/files/script-tmp-inta-_cra_del_pacu.pdf el 06/09/19.

Sarkar B., Tiwari G. N. (2006). Thermal modeling and parametric studies of a greenhouse fish pond in the Central Himalaya Region. pp. 3183.

Wisely B.Holliday J. E. and MacDonald R. (1981). Heating an Aquaculture pond with a solar pool blanket. pp. 385-387

Zhu S, Deltour J, Wang S. (1998). Modeling the thermal characteristics of greenhouse pond systems. Aquaculture Eng18, pp. 201-17. 